\title{
La inclusión de personas con diversidad funcional en la educación superior ${ }^{1}$ The inclusion of people with functional diversity in higher education
}

\author{
Carlos Fernando Chaves López ${ }^{2}$
}

\begin{abstract}
Resumen
Este artículo es una reflexión sobre la normatividad internacional y nacional de la inclusión de personas con diversidad funcional en la educación superior. La reflexión enfatiza en el derecho a la educación superior que tienen las personas con algún tipo de limitación y profundiza en los aspectos que deben trabajar las instituciones de educación superior para ser consideradas incluyentes. Finalmente, se hace un llamado a las universidades para que asuman el reto de comprometerse en la formación de las personas con diversidad funcional.
\end{abstract}

Palabras clave: Discapacidad; Derecho a la educación; Universidades; Inclusión.

\begin{abstract}
This article is a reflection about on international and national regulations on the inclusion of people with functional diversity in higher education. The reflection emphasizes the right to higher education that people with some type of limitation have, and it deepens the aspects that higher education institutions must work to be considered inclusive. Finally, a call is made to the universities to take up the challenge of committing to the training of people with functional diversity.
\end{abstract}

Keywords: Disability; Right to education; Universities; Inclusion.

\footnotetext{
${ }^{1}$ Artículo de reflexión.

${ }^{2}$ Mg. en Pedagogía. Docente de la Facultad de Ciencias Contables, Económicas y Administrativas de la Universidad Mariana. https://orcid.org/0000-0003-0338-1286 E-mail: cfchaves@umariana.edu.co

${ }^{*}$ Cómo citar este artículo: Chaves, C. (2018). La inclusión de personas con diversidad disfuncional en la educación superior. Hojas y Hablas, (16), 84-95. DOI:10.29151/hojasyhablas. n16a6
} 


\section{Introducción}

El derecho a la educación está consagrado en el artículo 26 de la Declaración de los Derechos Humanos (Naciones Unidas, ONU, s.f.), y en diversos tratados internacionales. Uno de los más recientes es el de la Convención sobre los Derechos de las Personas con Discapacidad (CRPD, por sus siglas en inglés), (ONU, 2008), firmada por los países miembros y ratificada por Colombia mediante la Ley 1346 de 2009.

La educación es también un derecho para las personas con diversidad funcional y no se limita a la formación preescolar, básica primaria, básica secundaria y media; debe extenderse a la educación superior. En consecuencia, se hace necesario que las universidades y los centros de formación superior asuman su papel en la protección de esta clase de personas y garanticen su acceso a la educación, rompiendo el esquema en el cual estos estudiantes deben adaptarse al sistema, y más bien, adaptar la universidad a los estudiantes, superando los obstáculos que les dificultan su acceso, permanencia y egreso satisfactorios.

\section{Deficiencia o discapacidad}

Los postulados de la CRPD (ONU y Naciones Unidas, Derechos Humanos, Oficina del Alto Comisionado, 2014), tienen como base el modelo social de relación entre comunidad y discapacidad. Este modelo, al decir de Palacios (2008), hace distinción entre los términos 'deficiencia' y 'discapacidad'.

El primero alude a la condición específica de un problema de funcionamiento en la mente o algún órgano del cuerpo, mientras el segundo se refiere a las limitaciones sociales que día a día experimentan las personas con alguna deficiencia. Es decir, la discapacidad no está en los individuos; es producto de las barreras sociales que los apartan y les dificultan su plena participación en la sociedad.

En este orden de ideas, el módulo 1 de la CRPD (ONU, 2008) indica que "las personas con discapacidad son objeto de conmiseración y dependen de la buena voluntad de la sociedad. [...] Carecen de empoderamiento, no controlan sus vidas y su participación es escasa o inexistente" (p. 9), además de ser consideradas una carga para la sociedad. Sus deficiencias físicas, mentales, intelectuales o sensoriales, les impiden participar plena y efectivamente en ella, en igualdad de condiciones respecto a las demás personas.

\section{El derecho a la educación superior de las perso- nas con diversidad funcional}

Los países firmantes de la CRDP, entre ellos Colombia, se adhieren a su articulado y específicamente, para el objeto de esta reflexión, al inciso 5 del artículo 24:

Los Estados partes asegurarán que las personas con discapacidad tengan acceso general a la educación superior, la formación profesional, la educación para adultos y el aprendizaje durante toda la vida, sin discriminación y en igualdad de condiciones con las demás. A tal fin, asegurarán que se realicen ajustes razonables para las personas con discapacidad. (Art. 24).

El nivel educativo de las personas con discapacidad, según el Consejo Nacional de Política Económica y Social (CONPES, 2013), es menor que el del resto de la población, y para el Departamento Administrativo Nacional de Estadísticas (DANE, 2010) y el Ministerio de Educación Nacional (MEN, 2013), el porcentaje de población en situación de discapacidad que ingresa a la educación superior, es inferior a $1 \%$. Este mínimo porcentaje se encuentra relacionado con prejuicios 
de las comunidades, instituciones académicas, familias, e incluso de las mismas personas con algún tipo de diversidad funcional.

En este contexto, y con miras a hacer efectivo el derecho a la educación superior de las personas con diversidad funcional, las instituciones deben empezar a considerar la inclusión de este tipo de alumnado en sus aulas, como tema prioritario, y comprometerse en la destinación del presupuesto y personal necesario para realizar las modificaciones arquitectónicas y la incorporación de contenidos, prácticas y servicios para esta población, que contribuyan a su desarrollo personal y social. Como afirman Martín-Padilla, Sarmiento y Coy (2013), "las personas con limitaciones físicas o mentales pueden desarrollar sus capacidades y potenciales, más allá de las creencias o perjuicios que pueda tenerse" (p. 195).

\section{Universidades inclusivas}

Según el Consejo Nacional de Acreditación (CNA) (2011) en Colombia, la educación superior es un servicio público que puede ser prestado por el Estado o por particulares, y tiene por objetivo la formación profesional. Las posibilidades para acceder a ella deberían estar disponibles para todas las personas, sin exclusión ni discriminación, si se tiene en cuenta las leyes promulgadas en Colombia y los acuerdos internacionales a los que la nación se ha adherido en este aspecto.

Se trata de un 'debería', porque a pesar de la legislación, son pocas las instituciones de educación superior (IES) que en el país han llevado a cabo acciones encaminadas a la inclusión de personas en condición de diversidad funcional, en las que se tenga en cuenta que estos alumnos, al igual que los regulares, son diferentes entre sí, así como sus discapacidades, y, por ende, sus necesidades. Por ello, para alcanzar una verdadera educación superior inclusiva, es requisito revisar las políticas institucionales bajo un enfoque diferencial que tenga en cuenta los distintos tipos de discapacidad. De este modo, se analiza a continuación aspectos en los que, a juicio del autor, deben trabajar las IES, si quieren ser incluyentes.

Accesibilidad: debe estar encaminada a detectar y eliminar las barreras que impiden el ingreso, partiendo de la hipótesis que todas las personas pueden aprender y el entorno educativo tendrá que ofrecer condiciones idóneas, tanto físicas como educativas. Para que esto se cumpla, en cuanto a la infraestructura, ha de trabajarse en la señalización, los accesos (rampas, ascensores), adecuación de las zonas comunes, baños, aulas, laboratorios, bibliotecas, entre otros. Respecto a las condiciones educativas, éstas pueden ser contempladas desde distintos ángulos: en primer lugar, tal como señala el inciso 4 del artículo 24 de la CRPD, las IES están llamadas a adoptar

Las medidas pertinentes para emplear a maestros, incluidos maestros con discapacidad, que estén cualificados en lengua de señas o Braille y para formar a profesionales y personal que trabajen en todos los niveles educativos. Esa formación incluirá la toma de conciencia sobre la discapacidad y el uso de modos, medios y formatos de comunicación aumentativos y alternativos apropiados, y de técnicas y materiales educativos para apoyar a las personas con discapacidad. (párr. 15).

Lo anterior implica igualmente, adaptaciones curriculares; es decir, en cuanto a los contenidos, competencias, niveles de desempeño, actividades, metodología y evaluación, con el objetivo de alcanzar un aprendizaje significativo de las personas con diversidad funcional. Así mismo, 
las condiciones educativas de accesibilidad deben considerar el uso de tecnologías y medios de comunicación, como instrumentos pedagógicos que coadyuven en la atención de las necesidades funcionales de los alumnos con necesidades educativas diversas. Frente a esto, las tendencias mundiales en educación promueven la inclusión y el impacto social a partir de la educación para todos como elemento integrador y de desarrollo humano. Esto se puede lograr mediante el uso de las tecnologías de la información y la comunicación (TIC), las cuales desempeñan un papel central en la flexibilidad curricular y en las adaptaciones pedagógico-didácticas, evidenciando el cambio que sugiere la sociedad del conocimiento y la información (Romero, 2017).

El mundo digital puede contribuir en el diseño y creación de ambientes de aprendizaje que tengan en cuenta la diversidad de alumnado, que innoven y potencien otras formas de enseñar, aprender, convivir e interactuar, y promuevan prácticas educativas orientadas a la igualdad, creando y motivando de esta manera la inclusión real y no en la forma como hoy en día se maneja (García y López, 2012). En este sentido, las universidades deben adelantar programas en los que la mayoría de asignaturas puedan ser cursadas de manera virtual a través de plataformas disponibles en la Web. Al respecto, Borda y Ormeño (2010) se refieren a la corriente pedagógica personalizada, la cual se construye de acuerdo con las particularidades de cada individuo, y la intención de formar personal con singularidad, autonomía, apertura, libertad y responsabilidad. Además, existe la corriente pedagógica social, encaminada a la evolución de la sociedad, buscando equidad y cooperación. De igual manera, la corriente pedagógica constructivista que considera que el cerebro edifica la experiencia y el conocimiento para posteriormente ordenarlos y darles forma.
Actualmente, según Rama (2013) las universidades no tienen prácticas discriminatorias de manera autónoma en relación con las personas con diversidad funcional, pero tampoco tienden a impulsar cambios significativos en sus dinámicas educativas que faciliten su acceso y permanencia. Una universidad accesible debe considerar mecanismos que garanticen la participación e interacción social de los estudiantes con necesidades educativas diversas, con los demás integrantes de la comunidad académica, dado que los organismos institucionales toman decisiones en derechos y deberes de todos sus integrantes.

Finalmente, la accesibilidad a la educación superior se encuentra vinculada con las facilidades económicas para ingresar, mantenerse y culminar estudios. Así, las directivas institucionales pueden ofrecer descuentos especiales, becas o algún auxilio para las personas con diversidad funcional. En Colombia, por ejemplo, la Fundación Saldarriaga Concha, en alianza con el Ministerio de Educación Nacional (MEN, 2012), la Fundación Carolina y el ICETEX, ofrece becas de pregrado y posgrado para personas con estas condiciones, de los estratos socioeconómicos 1, 2 y 3 de todo el país, quienes podrán acceder a becas de pregrado para realizar estudios técnicos, tecnológicos y universitarios en una IES de Colombia acreditada por el MEN. De igual forma, los profesionales con diversidad funcional se beneficiarán con becas para posgrados, cursos y estudios de maestría o doctorado en Estados Unidos. En España y Estados Unidos éstas cubren el valor de la matrícula, ayuda para alojamiento, manutención y seguro médico durante la estancia del becario en el respectivo país; incluyen tiquetes aéreos desde Bogotá, asesoría en los trámites de visa, apoyo para la instalación en el extranjero y ayuda para libros y materiales. 
Así mismo, la Universidad Nacional de Colombia, a través de sus políticas institucionales (Acuerdo 036 de 2012) establece la política institucional para la inclusión educativa de las personas con diversidad funcional. Para efectos de la aplicación de la prueba de admisión a programas curriculares de pregrado, se prevé ajustes para personas con discapacidad auditiva, discapacidad visual, discapacidad intelectual, discapacidad motora, discapacidad psicosocial, discapacidad sordo-ceguera y discapacidad múltiple.

Políticas institucionales: la formulación, puesta en marcha, seguimiento y evaluación permanente de los lineamientos en materia de educación superior para las personas con diversidad funcional, hará posible que los estudiantes en estas condiciones puedan ejercer sus derechos y cumplir con sus deberes. Todo ello debe basarse en el ordenamiento jurídico nacional, apoyarse en la autonomía institucional que por ley se otorga a las IES, así como en la Declaración Universal de los Derechos Humanos y en la normativa consagrada en la CDPR (2016).

Así, desde la Política Pública Nacional de Discapacidad e Inclusión Social (2014) se hace un llamado a todos los actores de la sociedad, para que participen en la construcción de oportunidades para el desarrollo de las potencialidades de las personas con diversidad funcional. De igual manera, en el marco de la CDPR, se insta a los Estados partes y a los miembros de sus sociedades a contribuir en el pleno desarrollo del potencial humano, la dignidad y autoestima de este grupo poblacional, objetivo al que pueden aportar de manera importante las IES. Esto puede llevarse a la práctica en la formulación de los planes de desarrollo, programas, proyectos institucionales, académicos-pedagógicos, de bienestar y reglamento estudiantil; estos deben ser planteados de tal forma que incluyan medidas antidiscriminatorias y promuevan la inclusión.

Conocimiento y formación en educación inclusiva: este elemento se refiere al compromiso que las universidades deben asumir respecto a la formación de docentes y a la evaluación de los prejuicios que sobre la diversidad funcional aún se evidencia en directivos, profesores y estudiantes. Según el Fondo de las Naciones Unidas para la Infancia (UNICEF, 2001), son escasas las IES que forman a los futuros profesores, que contemplan en el currículo asignaturas dirigidas a prepararlos para trabajar con personas en condición de diversidad funcional. En su mayoría, no se les instruye en adaptaciones curriculares o modalidades de evaluación, acordes con las necesidades educativas especiales.

Aunque se ha dado avances en la formación de profesores para la inclusión educativa, las universidades deberían esforzarse por incluir en su planta docente, maestros calificados y profesionales, como psicólogos, terapeutas, intérpretes, en aras de consolidar un grupo de trabajo interdisciplinario que responda a los requerimientos de los estudiantes con necesidades educativas diversas. Pero el trabajo debe ir más allá; debe incluir a los demás miembros de la comunidad educativa: familiares, compañeros y cuidadores, para que contribuyan a la consolidación de una universidad realmente inclusiva, sensibilizándolos y capacitándolos mediante actividades, proyectos, escuelas de padres y talleres.

\section{Discusión}

El concepto de 'diversidad funcional' debería darse en terreno neutral de la diferencia, con un sentido menos convencional de ésta última, lo cual podría ayudar a comprender las implicaciones positivas tanto en el campo educativo como 
en el social; por eso, es relevante tener conocimiento de la diversidad humana, para apreciar esas diferencias individuales que pueden llevarnos a comprender las diferencias grupales. De lo contrario ¿cómo podemos valorar que es la heterogeneidad, más que la homogeneidad, la que lleva a la supervivencia de las especies?

Integración e inclusión son dos conceptos básicos de la educación inclusiva. Entenderlos ayudará a encauzar el impacto en el rendimiento y adaptación de los estudiantes con necesidades educativas especiales (NEE), y, de paso, las creencias, percepciones, y actitudes del grupo familiar que les rodea, empezando por los padres y maestros.

La diversidad forma parte de la cultura y, en consecuencia, de la sociedad. Las nuevas tecnologías de la información están causando una verdadera revolución tecnológica y un proceso de globalización de diversas dimensiones (Gómez, 2001). Por tanto, se debe prestar mayor y más justa atención a su entendimiento y a las secuelas de no hacerlo, pues muchas diferencias pasarán a ser juzgadas y entendidas por lo que la sociedad cree que es correcto, y no por lo que realmente son. La diferencia se ha tratado como una problemática, lo cual ha llevado a tratar a las personas que difieren de las demás, como un 'déficit'.

Está surgiendo una sociedad mundial, un nuevo modelo de civilización ante el que las culturas tradicionales se sienten en peligro de extinción. ¿Es racional ese temor? Un sondeo aproximativo de la presencia de la diversidad cultural en las páginas de Internet sirve de punto de apoyo para desmentir la presunta homogeneización y las posiciones radicales contra la globalización. Ésta ofrece pluralidad de caras, constituye una realidad ambivalente, a la vez destructiva y creativa, llena de contradicciones, pero generadora de grandes oportunidades también para las culturas periféricas y minoritarias. (Gómez, 001, párr. 1).

Conviene analizar a profundidad la repercusión del trasfondo sociocultural en el origen de las diferencias. Las personas difieren en sus características. Las diferencias se hallan determinadas por el ambiente y son una construcción social. El problema está en considerar a la diferencia como una desventaja; de ahí que es imprescindible estudiar desde las distintas ciencias, la importancia de las creencias y acepciones culturales y sociales frente a este concepto.

En el campo educativo esas diferencias no pueden pasar desapercibidas: unos estudiantes responden con mayor rapidez al aprendizaje, asimilando los nuevos conceptos de manera más apropiada, generando tal vez una ventaja sobre los demás, pero es en el aula donde el papel del docente es relevante para saber encauzar esa diversidad y guiarles hacia el entendimiento común, de manera satisfactoria para el grupo en general, procurando evitar las brechas, aunque bien vale la pena preguntarse si existe una aptitud general para el aprendizaje (Snow, 1992).

En palabras de Cardona (2006), "los profesores deben evitar pensar en los alumnos, como inteligentes o no inteligentes, desde el momento en que hay muchas formas de ser inteligentes" (p. 23). Desafortunadamente, en muchas instituciones educativas solo se tiene en cuenta un conjunto limitado de aptitudes, especialmente las relacionadas con las matemáticas y la lógica, pero en cuanto se empiece a ampliar la percepción de inteligencia, se hará sin duda una gran contribución al desarrollo y formación de los estudiantes inteligentes; por tanto, los docentes deberán estar 
en capacidad de ofrecer y aceptar distintas maneras y caminos de realizar una actividad o una tarea, lo que equivale a decir que deben presentar sus contenidos de modos diversos, para favorecer el aprendizaje de un número más amplio de alumnos, teniendo en cuenta sus distintas competencias, más que su inteligencia.

Trasladándonos a la educación superior, es lógico suponer que al llegar a la universidad o a cualquier IES ya existe en la persona, una decisión frente a su vocación, guiada por sus potencialidades, aptitudes, intereses que muchas veces van de la mano con sus capacidades tanto físicas como mentales, pero no se puede desconocer que la globalización ha presionado para convertir a la educación en una mercancía. La competitividad se ha transferido a las aulas, mercantilizando la enseñanza y convirtiéndola en un negocio unificado que produce perfiles académicos en serie, en respuesta a los intereses del mercado, mas no a las necesidades de una buena educación, sin opciones que permitan generar diferencias o enriquecer el perfil; por tanto, los estudiantes se convierten en modelos profesionales estandarizados que solo responden a las necesidades del sistema educativo. Por eso la educación, especialmente la superior, debe trascender ese modelo mercantil y apostarle a una educación que reconozca los modos de la diversidad. Reconocernos en nuestras diferencias y diversidades, más allá del afán homogeneizante del movimiento global, es una de las grandes necesidades de nuestro tiempo, de tal manera que los valores y las culturas locales no sean sofocados, sino reconocidos.

Si bien para un profesor es difícil reconocer cada estilo de aprendizaje, sí debe ser consciente que cada estudiante tiene un estilo propio de enfrentar el problema, y debe procurar en lo posible, brindar un ambiente adecuado y tranquilo para lograr la apropiación de las ideas, habilidades, competencias, aptitudes y talentos. La manera como enfrente las diferencias repercutirá en su práctica pedagógica. Por tanto, debe prepararse para entender esa diversidad y contribuir a desmitificar esas ideas erróneas preconcebidas y estereotipadas que solo causan daño y mantienen la diferencia.

La raza, el estrato social, el género, las habilidades o la discapacidad, son características que traen consigo los niños al ingresar a un colegio. Muchas de ellas no cambiarán nunca y no pueden ser ignoradas, pero otras dependerán de un aprendizaje significativo y del modo como los docentes enfoquen la enseñanza, para encontrar sentido y placer al asistir a un aula. Con mayor razón cuando ingresen a la universidad, dado que es evidente el vínculo que se forja entre el mundo académico y el profesional. El respeto por la diversidad es algo que se debe implementar en los currículos de educación superior. Los estudiantes deben ser conocedores de la realidad social y cultural y se les debe enseñar a superar los etnocentrismos y prejuicios. La calidad de la docencia debe garantizar la preparación de unos profesionales competentes que se identifiquen con su quehacer y que adquieran competencias para su interacción con la sociedad, con vocación de servicio, pero también formados como seres humanos que practican sus valores y creencias en bien del otro. Entonces, queda por reflexionar en cómo los maestros debemos alcanzar los límites de conciencia y afrontar los nuevos horizontes.

Para ello se debe replantear las posturas educativas, y darles cabida a posibilidades eficientes y positivas en la educación que se imparte desde las propias experiencias, potenciando el diario quehacer pedagógico. De esta forma, estudiantes y maestros se transformarían en autores 
de su propia construcción de vida, reconociéndose a sí mismos en sus diferencias. Estos ámbitos no-determinados, "son justamente los espacios de construcción del sujeto; [...] si éste construye, la implicación de ese desafío es como capacitarlo para que lo haga" (Zemelman, 2009, p. 121).

El hecho de sentirse diferente interfiere en el aprendizaje $y$, en consecuencia, en el rendimiento y calidad de vida; no solo en el aula de clases, sino que tendrá repercusiones en su vida como adulto. Justamente por eso, para cualquier persona, no hay nada más importante que reconocer su identidad y tratar de preservarla para no aparentar lo que no se es. El futuro de muchas personas, instituciones y países depende de cómo se maneje la diversidad; de ahí la importancia de formarse en distintas áreas, siendo claros, críticos, responsables y justos.

Conocer y reconocer las características diversas que traen consigo los estudiantes ayuda a comprender su comportamiento y el del grupo o comunidad a la que pertenecen. Aunque tengan la misma edad, muchos difieren en su preparación, capacidad para aprender, sus experiencias, anhelos, contexto y circunstancias vitales, lo cual no significa de ningún modo, que sean menos inteligentes que otros que se adaptan con mayor facilidad al medio educativo. Y no importan esas diferencias, cuando los centros educativos crean un ambiente en el que todos se sienten respetados y aceptados, resaltando siempre el objetivo principal: desarrollar al máximo las capacidades de cada estudiante. El gran reto está en cómo agruparlos para que aprendan mejor y cómo enseñar de forma efectiva y práctica, de tal manera que se los prepare adecuadamente para enfrentar el mundo real.

La educación inclusiva de hoy hace que en las aulas sea necesario ajustar la enseñanza a las características heterogéneas de los estudiantes, lo cual requiere más ingenio por parte del docente y del sistema educativo, dado que todos deben sentirse apoyados y aceptados, en cuanto a sus necesidades educativas. Alumnos con NEE son aquéllos con inteligencia baja, o alta, con dificultades para el aprendizaje, o con discapacidades físicas, emocionales, sensoriales, que requieren atención especial. A su vez, la educación especial es el estudio diferencial de la educación, "cuando ese estudio recae sobre las personas con capacidades extremas por exceso o por defecto" (Jiménez, 1997, p. 77).

Fromm (2009) manifiesta que "la educación conduce con demasiada frecuencia a la eliminación de la espontaneidad y a la sustitución de los actos psíquicos originales por emociones, pensamientos y deseos impuestos desde afuera" (p. 278), y esto es justamente lo que debe evitar el docente de hoy. Por tal motivo, el desafío para los docentes es aprender a atender esos requerimientos, dándole un nuevo significado a su labor, modificando métodos, aprendiendo constantemente, actualizándose, ubicándose en posición de escucha y disposición de sorprenderse, porque

...aquellos que muestran su temor al cambio, a perder su estatus, su comodidad, no [se] arriesgan a soltar el poder y actúan desde la creencia del dominio y la subordinación, siendo prisioneros de sus miedos, sin darse opciones de actuar en libertad, como universo desconocido en las fronteras escolares. (Gómez, 2017, p. 16).

El concepto de inclusión va más allá del concepto de integración; sugiere que las personas sean valoradas por lo que son, más que por ser diferentes, para que puedan participar de cualquier 
actividad física, social, intelectual, funcional, las mismas que caracterizan a la sociedad común. Esto implica llevar a cabo políticas de reforma educativa que involucren cambios tanto en la didáctica escolar como en la ejecución de los derechos humanos, y para eso se requiere docentes comprometidos, con buena voluntad, con deseo de servir, capacitados y actualizados, que puedan generar y liderar un eficaz trabajo colaborativo. Hoy en día la educación inclusiva implica adoptar enfoques integrados que hagan énfasis en la motivación, el desarrollo neurológico, cognitivo, el ambiente, las necesidades educativas, de manera tal que se pueda intervenir en esta población, de manera exitosa.

\section{Conclusiones}

Los avances en Colombia relacionados con el derecho a la educación superior de las personas con diversidad funcional aún son incipientes, aunque la Constitución reconozca que todos los niños tienen derecho a la educación, aun aquellos con discapacidades físicas, cognitivas o de cualquier otro tipo. En esta materia han empezado a trabajar tan solo cinco universidades: Nacional, Pedagógica, de Antioquia, del Valle y del Rosario (Bernal, 2014). La cifra es desalentadora, si se tiene en cuenta que en el país hay más de 250 universidades, varias de ellas con sedes en distintos municipios, por lo que el número podría alcanzar incluso 300 , lo que permite afirmar que solo 1,3\% de las IES han asumido su responsabilidad frente a los estudiantes con algún tipo de diversidad funcional.

Queda entonces mucho por hacer y se debe iniciar por modificar los imaginarios sociales sobre la discapacidad y erradicar los prejuicios hacia este grupo poblacional, para que el ejercicio de su derecho a la educación superior sea una realidad. Podría empezarse por cambiar el término 'discapacidad', por el de 'diversidad funcional', pues éste dignifica, humaniza y lleva a reconocer las potencialidades de quienes tienen algún tipo de deficiencia o limitación, aunque la realidad no se modifica porque se cambie una palabra ni aun incluso porque se legisle en un sentido u otro, si no hay verdaderos cambios en la cultura, las actitudes, los comportamientos, la preparación.

Esto es relevante en la era de la globalización, caracterizada porque día a día hay más avances científicos y tecnológicos que tienen en común la universalidad económica, cultural, medioambiental y la reivindicación de los derechos humanos mediante manifestaciones individuales y grupales que llaman a la movilización de la sociedad hacia el acceso equitativo de todos sus integrantes a los bienes y servicios que ésta ofrece. Uno de ellos, quizás el más importante, es la educación, dado que puede considerarse como la mejor herramienta para edificar una sociedad mundial que prepare a todos sus integrantes como individuos autónomos y conscientes de cuánto pueden contribuir al bienestar colectivo desde su papel en los contextos en los que se desenvuelven.

Es por ello que las universidades están obligadas a comprometerse y a responder al reto que la sociedad globalizada les propone frente a las personas con diversidad funcional. El llamado es entonces a trabajar en accesibilidad, políticas institucionales y conocimiento y en la formación en educación inclusiva. La inclusión, además, debe hacerse extensiva a la vinculación laboral de docentes y empleados con diversidad funcional, dado que la universidad no es tan solo un espacio de interacción entre profesores y alumnos, sino un lugar donde confluyen otros, tales como personal administrativo y de servicios que, con su labor, otorgan continuidad al quehacer institucional. 
De modo que, docentes bien formados, sin prejuicios y actualizados, constituyen una garantía de una enseñanza calificada. Estudiantes tratados en un ambiente de respeto se convierten en referentes de que es posible lograr un aprendizaje bueno y significativo. Si la preocupación de estos dos aspectos es constante y verdadera, se estará trabajando en una reforma de la educación que posibilite una real educación para la diversidad.

En países como Colombia, tener una diversidad funcional puede reducir grandemente las posibilidades de educarse apropiadamente; muchos de esos niños con necesidades educativas diversas se ven enfrentados a problemas de movilidad que pueden agravar su situación.

Por encima de cualquier diferencia, se debería enseñar a aprender, a argumentar, a criticar, que son tareas que el sistema educativo y el profesor deben cumplir para iniciar la búsqueda del conocimiento. En Colombia hay una marcada diversidad, y los estudiantes han dejado de ser homogéneos; el profesor ya no puede trabajar ignorando los contrastes en habilidad, valores, experiencia, personalidad, cultura e incluso idioma, en cualquier salón de clases; por ello necesita conocer y reconocer los saberes, talentos, capacidades y posibilidades de los estudiantes universitarios, máxime cuando serán ellos los futuros profesionales encargados, desde su rama, de tomar las riendas de la sociedad.

Para finalizar, se considera importante proponer un trabajo interinstitucional en el que participen universidades públicas y privadas, junto con los estamentos gubernamentales, a fin de promover la inclusión educativa superior para las personas con diversidad funcional. Para crear las condiciones idóneas y necesarias en educación inclusiva, no solo se requiere buena actitud, sino un cambio de mentalidad que incluya todo el sistema educativo. Es urgente una nueva forma de pensar y concebir las diferencias, lo que lleva como consecuencia, a una nueva forma de entender la educación.

\section{Referencias}

Bernal, A. (2014). Universidad e inclusión de personas con discapacidad. Paidea, (19), 55-61.

Borda, J. y Ormeño, M. (2010). Las corrientes pedagógicas contemporáneas y los estilos de enseñanza en la educación física. Investigación Educativa, 14(26), 79-104.

Cardona, M. (2006). Diversidad y educación inclusiva. Madrid, España: Pearson Educación S.A.

Congreso de la República de Colombia. (31 de julio de 2009). Ley 1346 "Por medio de la cual se aprueba la 'Convención sobre los Derechos de las personas con Discapacidad', adoptada por la Asamblea General de la Naciones Unidas el 13 de diciembre de 2006”. Bogotá, Colombia. Recuperada de http://www.alcaldiabogota.gov.co/sisjur/ normas/Norma1.jsp?i=37150

Consejo Nacional de Acreditación (CNA). (2011). El sistema de educación superior en Colombia. Recuperado de https://www.cna. gov.co/1741/article-187279.html

Consejo Nacional de Política Económica y Social (CONPES). (2013). Política Pública Nacional de Discapacidad e Inclusión Social. Recuperado de https://colaboracion.dnp.gov. co/CDT/Conpes/Social/166.pdf

Convención sobre los derechos de las personas con discapacidad. (2016). Recuperado de https://www.1decada4.es/mod/page/view. php?id=270 
Departamento Administrativo Nacional de Estadísticas (DANE). (2010). Discapacidad. Recuperado de https://www.dane. gov.co/index.php/estadisticas-por-tema/ demografia-y-poblacion/discapacidad

Fromm, E. (2009). El miedo a la libertad (3a. ed.). Barcelona, España: Paidós Ibérica.

García, M., y López, R. (2012). Explorando, desde una perspectiva inclusiva, el uso de las TIC para atender a la diversidad. Profesorado, Revista de currículum y formación del profesorado, 16(1). Recuperado de http://www. ugr.es/ recfpro/rev161COL4.pdf

Gómez, P. (2001). Evolución de la diversidad cultural en la sociedad global informacional. Recuperado de http://www.ugr.es/ pwlac/ G17_02Pedro_Gomez_Garcia.html

Gómez, C. (2017). Reconocimientos de las diversidades en maestras de Enfermería de la Universidad Mariana a partir de sus procesos de enseñanza-aprendizaje (Tesis de Maestría). Universidad de Manizales. Recuperado de http://ridum. umanizales.edu.co:8080/xmlui/bitstream/ handle/6789/3175/TESIS\%20CRISLY\%20 MARICELA\%20GOMEZ\%20LEGARDA. pdf ? sequence $=2$ \&isAllowed $=y$

Fondo de las Naciones Unidas para la Infancia. (UNICEF). (2001). Inclusión de niños con discapacidad en la escuela regular. Recuperado de http://www.unicef.cl/archivos_documento/47/debate8.pdf

Jiménez, C. (1997). Pedagogía diferencial. Madrid, España: UNED.

Martín-Padilla, E., Sarmiento, P. y Coy, L. (2013). Educación inclusiva y diversidad funcional en la universidad. Revista de la Facultad de Medicina de la Universidad Nacional, 61(2), 195-204.

Ministerio de Educación Nacional (2012) Becas para personas con discapacidad.
Recuperado de https://www.mineducacion. gov.co/observatorio/1722/article-305424. html

Ministerio de Educación Nacional (MEN). (2013). Panorama de la inclusión en la Educación Superior en Colombia. Recuperado de https://www.mineducacion.gov.co/ cvn/1665/w3-article-327505.html

Ministerio de Salud y Protección Social. (2014). Política Pública Nacional de Discapacidad e Inclusión Social 2013-2022. Recuperado de https://www.minsalud.gov.co/sites/rid/ Lists/BibliotecaDigital/RIDE/DE/PS/politica-publica-discapacidad-2013-2022.pdf

Naciones Unidas. (s.f.). La Declaración Universal de los Derechos Humanos. Recuperado de http://www.un.org/es/ universal-declaration-human-rights/

Naciones Unidas y Naciones Unidas, Derechos Humanos, Oficina del Alto Comisionado. (2014). Convención sobre los Derechos de las Personas con Discapacidad. Recuperado de https://www.ohchr.org/Documents/ Publications/AdvocacyTool_sp.pdf

Palacios, A. (2008). El modelo social de discapacidad: orígenes, caracterización y plasmación en la Convención Internacional sobre los Derechos de las Personas con Discapacidad. Madrid, España: Grupo Editorial Cinca.

Rama, C. (2013) La educación virtual como la modalidad educativa para las personas con necesidades especiales: solo en la red no hay personas con discapacidad. Recuperado de http://www.redalyc.org/ html/1891/189126039016/

Romero, W. (2017). La Educación Virtual como una Alternativa para la Inclusión de Personas en Condición de Diversidad Funcional: Un Análisis de la Educación Superior 
del Futuro Recuperado de https://stadium. Zemelman, H. (2009). Uso crítico de la teoría. En unad.edu.co/preview/UNAD.php?url=/ torno a las funciones analíticas de la totalibitstream/10596/14337/1/18130913.pdf

Snow, R. (1992). Aptitude Theory: yesterday, todad (2a. ed.). México: Instituto Politécnico day and tomorrow. Educational PsycholoNacional. gist, 27(1), 5-32.

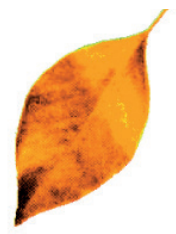

